\title{
Extracorporeal Shock Wave Therapy (ESWT) Versus Local Steroids Injection in the Management of Carpal Tunnel Syndrome
}

\author{
Gehad Sweilam, Mohsen Elshahaly* and Mohamed Hefny \\ Department of Physical medicine and Rehabilitation, Egypt
}

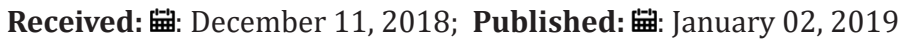

*Corresponding author: Mohsen Elshahaly, Department of Physical medicine and Rehabilitation, Egypt

\begin{abstract}
Aim of the Work: to evaluate the efficacy of Extracorporeal Shock Wave Therapy (ESWT) in the management of carpal tunnel syndrome (CTS) and compare it with local steroid injection.

Patients and Methods: 53 patients diagnosed with CTS successfully completed the study. Eleven patients were excluded due to non-compliance with study visits. Patients were randomized into two groups: a control group made up of 28 patients injected with one milliliter of Triamcinolone Acetonide (40mg) and a study group of 25 patients received two sessions of ESWT with one-week in-between of 2500 shocks at a frequency of $10 \mathrm{~Hz}$ and intensity of 2 bars (BLT-5000 SWT power). Patients were assessed at baseline then after 2 and 4 weeks using VAS score, electrophysiological studies and Boston Carpal tunnel questionnaire (BCTQ) score.

Results: Both groups were similar in age, sex distribution, and duration of symptoms at baseline. There was a significant improvement of symptoms assessed by pain VAS score and BCTQ score in both groups during follow-up. Nerve conduction studies of median nerves showed significant decrease of distal motor latencies and increase of amplitude in both groups after 2 and 4 weeks. Comparing both groups, there was no difference in pain VAS and BCTQ scores, distal motor latency and nerve conduction velocity of median nerves between both groups on the second and third visits.
\end{abstract}

Conclusion: ESWT is as effective as local steroid injections in the management of CTS but ESWT is better being non-invasive.

Keywords: Carpal tunnel syndrome; ESWT; extracorporeal shockwave therapy; Boston's carpal tunnel questionnaire; nerve conduction study

Abbreviations: CTS: Carpal Tunnel Syndrome; ESWT: Extracorporeal Shock Wave Therapy; BCTQ: Boston Carpal Tunnel Questionnaire; CS: Cortico Steroid; NSAIDs: Non-Steroidal Anti-Inflammatory Drugs; VAS: Visual Analog Scale; ANOVA : Analysis of variance

\section{Introduction}

Carpal tunnel syndrome (CTS) is a combination of characteristic symptoms and signs that occur following compression of the median nerve within the carpal tunnel [1]. Paranesthesia and/ or pain either mainly at night or sometimes the whole day in the median nerve distribution such as numbness and falling of objects out of the patients' hand are characteristic [2]. Patients may have impaired sensation to pinprick and/or light touch in the lateral three digits and sometimes may have impaired sensation in the lateral aspect of the fourth digit. When CTS is chronic and severe, it leads to wasting of the thenar muscles [3]. Conservative treatment for CTS includes splinting of the wrist in neutral position using cock-up splint in addition to the use of oral non-steroidal antiinflammatory drugs (NSAIDs) for pain relief [4]. In cases of mild to moderate CTS, corticosteroid (CS) injections beneath the transverse carpal ligament are the preferred treatment option. CS injections are administered to reduce the inflammation and swelling of the soft tissues around the median nerve (e.g., the flexor tenosynovium) and thereby to reduce the pressure on median nerve [5]. Local CS injections are widely used in CTS treatment. Injected dose ranges between 15-40mg of triamcenolone acetate. Site of injection is just medial to the tendon of the palmaris longus at a distance ranging from 0 to $4 \mathrm{~cm}$ proximal to the proximal crease of the wrist [6].

Shock waves are defined as a sequence of single sonic pulses characterized by a high peak pressure $(100 \mathrm{MPa})$, fast pressure rises $(<10 \mu \mathrm{secs})$, and short duration $(10 \mu \mathrm{secs})$ [7] .Shockwaves are not aimed to be strong enough to destroy tissues. Its strength is adjusted to have biological effects on interstitial tissues leading to tissue healing and regeneration. Shockwaves were used to treat 
enthesopathies and tendinopathies through stimulation of tissue repair and through their analgesic effects. ESWT is thought to heal through different mechanisms including neovascularization, differentiation of mesenchymal stem cells and local release of angiogenetic factors [8]. The effectiveness of ESWT for the treatment of entrapment neuropathies, including CTS is not well investigated. Few studies suggested that ESWT was effective in the treatment of CTS, yet their results were controversial. This study was designed to evaluate the effectiveness of ESWT for the management of CTS and to compare its efficacy against that of local CS injection.

\section{Methods}

This randomized study was carried out in the Rheumatology and Rehabilitation outpatient clinic in Suez Canal University Hospital between May 2015 and October 2016. A total of 64 patients with clinical and electrophysiological diagnosis of CTS were enrolled. We excluded patients with other diseases such as diabetes mellitus, peripheral neuropathies and concomitant lower limbs symptoms. Patients were randomized into two groups; a study group receiving ESWT consisting of 32 patients and a control group of 32 subjects receiving local steroids injection. At baseline, all patients in both groups were evaluated by full medical history, physical examination and electrophysiological studies then marked the level of pain on visual analog scale (VAS) $(0-10)$, where 0 is pain-free and 10 is the worst imaginable pain. Patients' clinical symptoms were evaluated using Boston's carpal tunnel questionnaire (BCTQ) symptoms severity score. Patients were assessed by motor nerve conduction studies (MNCS) of both Median and Ulnar nerves through comparing their distal terminal motor latencies (DML) at baseline and on each visit. Patients were included in the study if median nerve DML exceeded 4.5 milliseconds (ms).

The participants were instructed to avoid using any other treatments including anti-inflammatory medications during the study. For the control group, needle was inserted at the proximal wrist crease, just ulnar to palmaris longus tendon, at a 30degree angle to the skin and aiming towards the index finger. One milliliter of Triamcinolone acetonide ( $40 \mathrm{mg}$ ) was injected into the vicinity of the median nerve once at baseline. For the study group, patient's forearm was placed on a table with the palm facing upwards. ESWT target site was located over the palmar aspect of the wrist joint (between tendons of flexor carpi radialis and Palmaris longus).

Each patient received two sessions of ESWT with one-week interval that comprised 2500 shocks at a frequency of $10 \mathrm{~Hz}$ and intensity of two-bars (BLT-5000SWT power). The probe was positioned perpendicular on the patient's palm, and ultrasound gel was used as a coupling agent. After 2 and 4 weeks, patients were evaluated by pain VAS, BCTQ and MNC studies of median nerve.

\section{Statistical Analysis}

Data were processed using SPSS version 17 (SPSS Inc., Chicago, IL, USA). Quantitative data were expressed as means \pm SD and qualitative data were expressed as numbers and percentages. Analysis of variance (ANOVA) followed-up by Tukey's multicomparison analysis procedure was used to test significance of difference for quantitative variables, while student t-test was used to compare both groups. A p-value $<0.05$ was considered statistically significant.

\section{Results}

A total of 64 patients were enrolled into the study. Out of them, 11 patients were excluded due to non-compliance with the study visits. The following analyses excluded these patients. 53 patients finished the study successfully. Subjects who finished the study were 9 males (16.9\%) and 44 females (83.1\%). ESWT group consisted of 25 patients and steroid group of 28 patients. Both groups were comparable in age, sex, and duration of symptoms. The mean \pm SD age of patients was $37.6 \pm 8.5$ years old for ESWT group and $36.8 \pm 8.8$ years old for the steroid group. There was no difference between both groups regarding duration of symptoms (25.1 \pm 19.3 months for ESWT group versus 26.4 \pm 17.9 months for steroid group). The pain VAS score for the ESWT group was (mean \pm SD) $8.46 \pm 1.6$ at baseline and there was a significant improvement after 2 and 4 weeks ( $3.2 \pm 1.9$ and $1 \pm 1.38$ respectively) respectively as shown in Figure 1a. Patients within the steroid group showed similar results to the ESWT group, with pain VAS score of (mean \pm SD) $9.3 \pm 1.2$ at baseline and there was a significant improvement after 2 and 4 weeks ( $3.4 \pm 1.9$ and $0.96 \pm 1.5$ respectively) as shown in Figure $1 \mathrm{~b}$. We compared the change in pain VAS score between both groups at each visit. There was no significant difference between both groups. Regarding BCTQ score, ESWT group showed mean \pm SD of $34.3 \pm 7.2$ at baseline. There was a significant improvement after 2 and 4 weeks $15.1 \pm 3.9$ and $12.3 \pm 2.1$ consecutively as shown in Figure 2a. Local steroid group subjects showed similar results to ESWT group, with a mean \pm SD of $36.1 \pm 6.5$ at baseline and showing significant improvement after 2 and 4 weeks $(16.3 \pm 5.8$ and 12.6 \pm 3.1 ) as shown in Figure $2 \mathrm{~b}$.

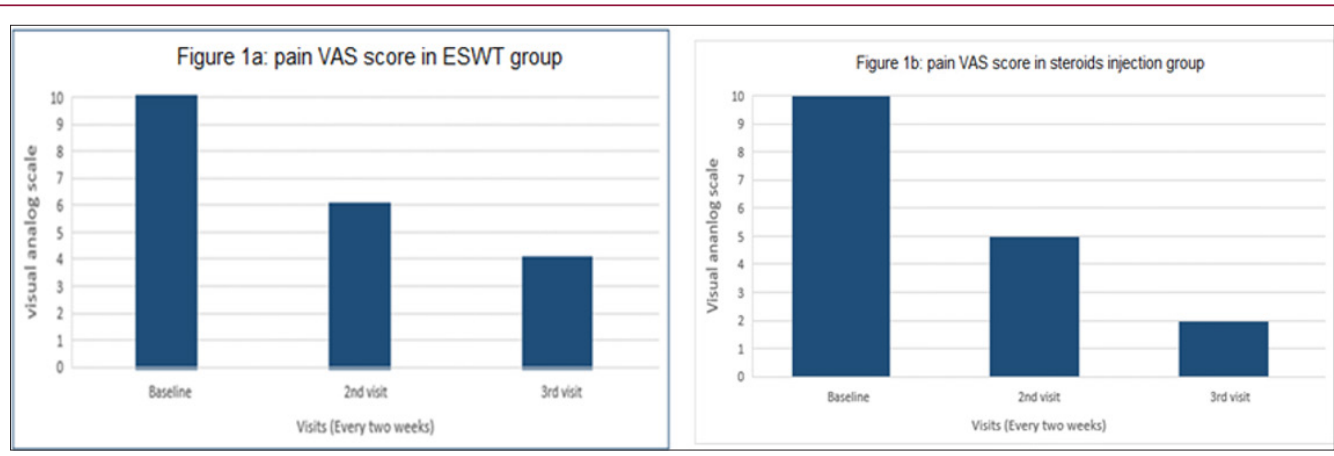

Figure 1: Showing changes in pain VAS at different timepoints. 


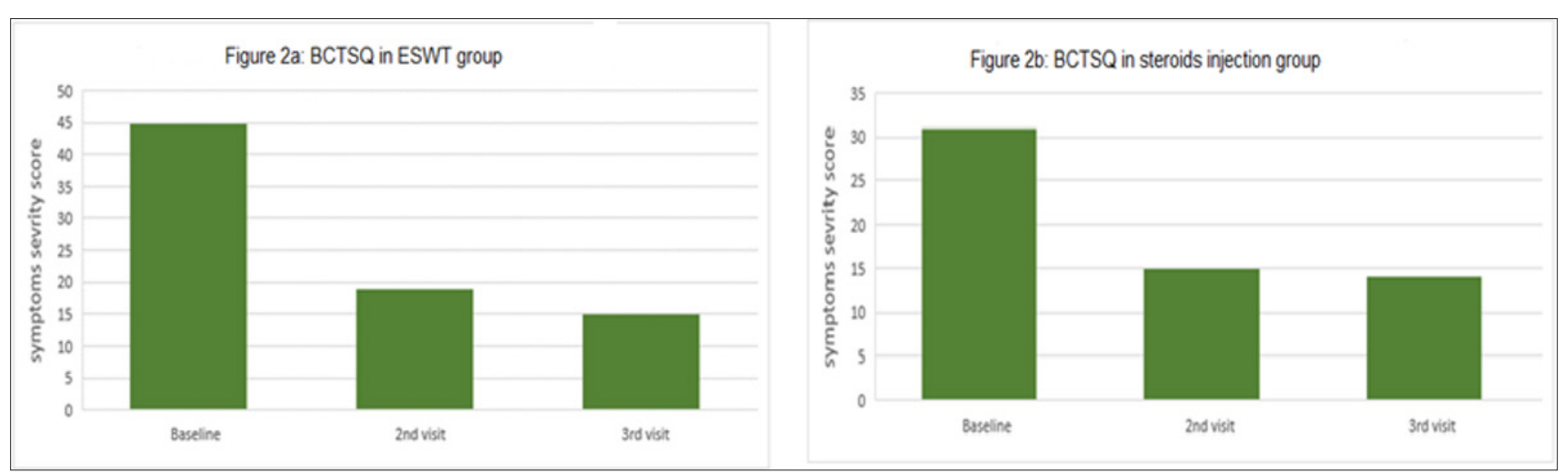

Figure 2: Showing changes in BCTQ scores at different timepoints.

Table 1: Showing the baseline characteristics of patients in both groups.

\begin{tabular}{|c|c|c|}
\hline & $\begin{array}{c}\text { ESWT Group } \\
(\mathbf{n = 2 5 )}\end{array}$ & $\begin{array}{c}\text { Steroid Injection } \\
\text { Group }(\mathbf{n = 2 8})\end{array}$ \\
\hline Age (Years), mean $\mathbf{\pm S D}$ & $37.6 \pm 8.5$ & $36.8 \pm 8.8$ \\
\hline Sex & & \\
\hline Male & 4 & 5 \\
\hline Female & 21 & 23 \\
\hline $\begin{array}{c}\text { Symptoms Duration } \\
\text { (Months) (Mean } \mathbf{\text { SD) }}\end{array}$ & $25.1 \pm 19.3$ & $26.4 \pm 17.9$ \\
\hline
\end{tabular}

Table 2: Showing NCS results at different timepoints.

\begin{tabular}{|c|c|c|}
\hline & $\begin{array}{c}\text { ESWT } \\
\text { Group }\end{array}$ & $\begin{array}{c}\text { Steroid Injection } \\
\text { Group }\end{array}$ \\
\hline DML of median nerve, $\mathrm{m}$ secs & & \\
\hline Baseline & $4.9 \pm .456$ & $5.29 \pm .96$ \\
\hline 2 wks. & $4.25 \pm .54$ & $4.58 \pm .96$ \\
\hline 4 wks. & $3.9 \pm .57$ & $4.13 \pm .86$ \\
\hline mV & & \\
\hline Baseline & $10.76 \pm 2.8$ & $10.42 \pm 3.7$ \\
\hline 2 wks. & $11.69 \pm 2.6$ & $10.53 \pm 3.4$ \\
\hline 4 wks. & $12.36 \pm 2.6$ & $11.3 \pm 3$ \\
\hline Baseline & & \\
\hline 2 wks. & $58.3 \pm 4.84$ & $56.7 \pm 4.22$ \\
\hline NCV of median motor nerve, $\mathbf{m} / \mathbf{s e c}$ & $57.12 \pm$ & $58 . \pm 5.47$ \\
\hline 4 wks. & 3.83 & \\
\hline & $58.37 \pm$ & $60.04 \pm 5.49$ \\
\hline
\end{tabular}

We compared the change in BCTQ score between both groups at each visit. There was no significant difference between both groups. Distal terminal motor latencies showed significant improvement during follow-up in both groups. In ESWT group, terminal latencies showed significant improvement with a latency of $4.9 \pm 0.5 \mathrm{~ms}$. (at baseline), $4.3 \pm 0.5 \mathrm{~ms}$. (second visit) and $3.9 \pm 0.57 \mathrm{~ms}$. (third visit). Regarding the local steroid injection group, terminal latency was $5.29 \pm 0.96$ (at baseline) then $4.58 \pm 0.96 \mathrm{~ms}$. and $4.13 \pm 0.8 \mathrm{~ms}$. after 2 and 4 weeks respectively. Furthermore, there was a significant increase in amplitude in the ESWT group during follow-up visits.
At baseline, amplitude was $10.8 \pm 2.8 \mathrm{mV}$. After 2 and 4 weeks, amplitude was $11.7 \pm 2.6 \mathrm{mV}$ and $12.4 \pm 2.6 \mathrm{mV}$ respectively. In the local steroid injection group, there was no significant change in amplitude after 2 weeks $10.4 \pm 3.7 \mathrm{mV}(\mathrm{p}=0.775)$. However, there was a significant improvement in amplitude between the first and third visits $10.4 \pm 3.7 \mathrm{mV}$ and $11.3 \pm 3 \mathrm{mV}$ (Tables $1 \& 2$ ).

The conduction velocity (CV) of median nerves did not change over the three visits in the ESWT group $58.3 \pm 4.8 \mathrm{~m} / \mathrm{s}, 57.1 \pm 3.8 \mathrm{~m} / \mathrm{s}$ and $58.4 \pm 3.8 \mathrm{~m} / \mathrm{s}$. Similarly, patients from the steroid group showed no significant difference in CV between the first and second visits $56.7 \pm 4.2 \mathrm{~m} / \mathrm{s}$ and $58 \pm 5.5 \mathrm{~m} / \mathrm{s}(\mathrm{p}=0.169)$. Though, there was a small but significant improvement between the first and third visits, CV increased from $56.7 \pm 4.2 \mathrm{~m} / \mathrm{s}$ to $60 \pm 5.5 \mathrm{~m} / \mathrm{s}$. By comparing the pain VAS, BCTQ scores, distal motor latencies and nerve conduction velocities of both median nerves at the third visit, there was no significant difference between both groups.

\section{Discussion}

In ESWT group, there was a significant decrease in pain VAS score after one month by $74 \%$ from baseline, and BCTQ score decreased by $40 \%$ after one month from baseline. A similar study investigated the effect of one session of ESWT of 1000 shocks at a frequency of 360 shocks per minute; the energy level was set at the maximum level tolerated by the patient $(0.09 \sim 0.29 \mathrm{~mJ} /$ $\mathrm{mm} 2$ ). There was a significant improvement of pain VAS score by $25 \%$ and BCTQ score by $20.2 \%$. However, there was no significant improvement in electrodiagnostic studies of the median nerve in ESWT group [7]. In the present study, the DML showed significant reduction in the ESWT group changing from $4.9 \pm 0.456 \mathrm{~ms}$. at baseline to $4.25 \pm 0.54 \mathrm{~ms}$. after 2 weeks and $3.9 \pm 0.57 \mathrm{~ms}$. after four weeks. Another study assessed the effectiveness of therapeutic ultrasound, cryo-ultrasound and ESWT in improving pain and function in patients with CTS. They showed that the three groups had improvement in pain VAS and BCTQ scores. However, Patients in ESWT group showed greater pain improvement at 12-weeks follow-up when compared with the other groups. Though, neurophysiological assessment was not done in the study [9].

Another study assessing the effect of ESWT on CTS showed a significant improvement in the pain VAS, BCTQ scores, and mean cross-sectional area (CSA) of the median nerve in the intervention group throughout the study as compared to the control group 
(except for BCTQ severity at week 12 and CSA at weeks 1 and 4) [10].

The exact mechanism by which ESWT can affect the nerve fiber is not fully understood. A study investigated if ESWT accelerates the regeneration of injured nerves in a rat model. They showed that ESWT induces axonal regeneration. This probably occurs through a faster Wallerian degeneration and removal of degenerated axons. This may help the injured axons to heal [11]. This might be explained by the release of nitric oxide in the injured nerve leading to improved short-term anti-inflammatory effect and long-term tissue regeneration effect [12].

\section{Conclusion}

ESWT is as effective as local steroids injection for management of CTS but ESWT is better being noninvasive. However, larger longterm studies are needed to confirm these results.

\section{References}

1. Fernandez de Las Penas C, Fernandez Munoz JJ, Navarro Pardo E, Da Silva Pocinho RF, Ambite Quesada S, et al. (2016) Identification of Subgroups of Women with Carpal Tunnel Syndrome with Central Sensitization. Pain medicine Malden, Mass 17(9): 1749-1756.

2. Kandil MR, Darwish ES, Khedr EM, Sabry MM, Abdullah MA (2012) A community-based epidemiological study of peripheral neuropathies in Assiut, Egypt. Neurological research 34(10): 960-966.

3. Ibrahim I, Khan WS, Goddard N, Smitham P (2012) Carpal tunnel syndrome: a review of the recent literature. Open Orthop J 6: 69-76.

4. O Connor D, Marshall S, Massy Westropp N (2003) Non-surgical treatment (other than steroid injection) for carpal tunnel syndrome. Cochrane Database Syst Rev 2013(1): CD003219.

ISSN: 2574-1241

DOI: 10.26717/BJSTR.2019.12.002281

Mohsen Elshahaly. Biomed J Sci \& Tech Res

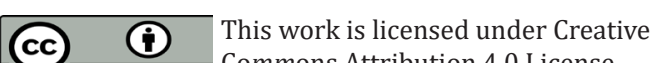

Submission Link: https://biomedres.us/submit-manuscript.php
5. Marshall S, Tardif G, Ashworth N (2007) Local corticosteroid injection for carpal tunnel syndrome Cochrane. Database Systc Rev 2007(2): CD001554.

6. EL Badawy, MAA F (2015) Electrophysiological and clinical comparison of local steroid injection by means of proximal versus distal approach in patients with mild and moderate carpal tunnel syndrome. Egyptian Rheumatology and Rehabilitation 42(3): 120-127.

7. Seok H, SH Kim (2013) The effectiveness of extracorporeal shock wave therapy vs. local steroid injection for management of carpal tunnel syndrome: A randomized controlled trial. American Journal of Physical Medicine \& Rehabilitation 92(4): 327-334.

8. Notarnicola A, B Moretti (2012) The biological effects of extracorporeal shock wave therapy (eswt) on tendon tissue. MLTJ Muscles, Ligaments and Tendons Journal 2(1): 33-37.

9. Paoloni M, Tavernese E, Cacchio A, D Orazi V, Ioppolo F, et al. (2015) Extracorporeal shock wave therapy and ultrasound therapy improve pain and function in patients with carpal tunnel syndrome. A randomized controlled trial. European journal of physical and rehabilitation medicine 51(5): 521-528.

10. Wu YT, Ke MJ, Chou YC, Chang CY, Lin CY, et al. (2016) Effect of radial shock wave therapy for carpal tunnel syndrome: A prospective randomized, double-blind, placebo-controlled trial. Journal of orthopedic research : official publication of the Orthopedic Research Society 34(6): 977-984.

11. Hausner T, Pajer K, Halat G, Hopf R, Schmidhammer $R$, et al. (2012) Improved rate of peripheral nerve regeneration induced by extracorporeal shock wave treatment in the rat. Experimental Neurology 236(2): 363-370.

12. Mariotto S, Cavalieri E, Amelio E, Ciampa AR, de Prati AC, et al. (2005) Extracorporeal shock waves: from lithotripsy to anti-inflammatory action by NO production. Nitric Oxide 12(2): 89-96.

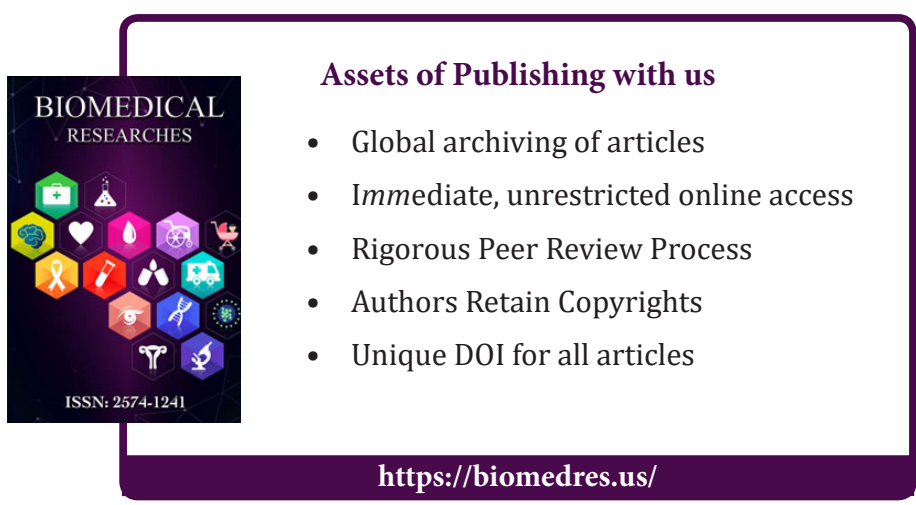

